\title{
Pregnancy (white) matters
}

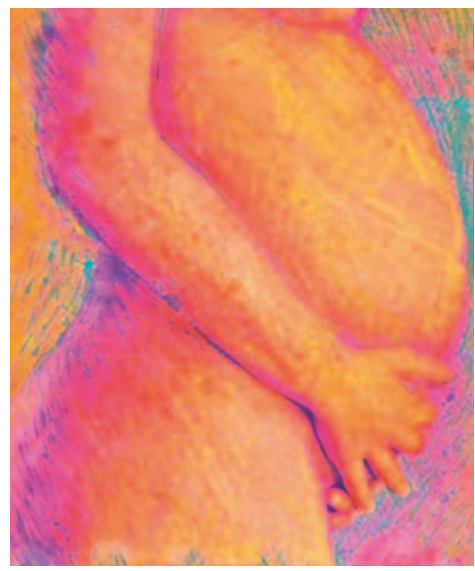

Multiple sclerosis (MS) is a chronic inflammatory disease that destroys the myelin sheath of neurons, leading to progressive loss of sensation, memory and movement. A Canadian research group have now uncovered the agent responsible for the remission of MS during pregnancy, offering hope for new treatments that could reverse, not just alleviate, the symptoms.

The clinical improvement of patients with MS during pregnancy is well documented, but the mechanisms responsible for it are poorly understood. Pregnancy-induced changes to the immune system have been suggested to mediate MS remission, but Weiss and colleagues show that axon remyelination is also involved. The authors found a remarkable increase in the proliferation of oligodendrocyte precursor cells in the corpus callosum and spinal cord of pregnant mice. The newly generated oligodendrocytes matured during the postpartum period, and this correlated with an increased number of myelinated axons in the maternal CNS 14 days postpartum. Furthermore, when myelin lesions were chemically induced both lesion size and the proportion of demyelinated axons were reduced in pregnant mice compared with virgin ones. BrdU tracing and quantitative analysis of lesioned axons revealed that these effects were primarily attributable to enhanced remyelination rather than neuroprotection during pregnancy.

As pregnancy-induced oligodendrocyte proliferation coincides with gestational increases in the proinflammatory hormone prolactin (PRL), the authors investigated whether PRL signalling prompted oligodendrocyte precursor cell division. They found that PRL promotes the generation of new oligodendrocytes in vitro, and that pregnancy-enhanced oligodendrocyte generation was reduced in $\mathrm{Prl}^{+/-}$mice. Moreover, PRL administration increased the number of oligodendrocytes in virgin mice and mimicked the effects of pregnancy on myelination when white matter damage was induced.

These findings suggest that PRL helps reverse the symptoms of MS during pregnancy by repairing myelin damage. Demyelination not only contributes to MS, but is also a hallmark of other neurological disorders such as spinal cord injury, stroke and age-related dementia. The possibility of harnessing the ability of PRL to repair myelin damage, while suppressing its proinflammatory effects in non-pregnant patients, holds promise for future therapeutic applications.

Monica Hoyos Flight

ORIGINAL RESEARCH PAPER Gregg C. et al White matter plasticity and enhanced remyelination in the maternal CNS.J. Neurosci. 27, 1812-1823 (2007) 